\title{
AN EFFICIENT INTRUSION DETECTION SYSTEM WITH CUSTOM FEATURES USING FPA-GRADIENT BOOST MACHINE LEARNING ALGORITHM
}

\author{
D.V. Jeyanthi ${ }^{1}$ and Dr. B. Indrani ${ }^{2}$ \\ ${ }^{1}$ Assistant Professor, Department of Computer Science, \\ Sourashtra College, Madurai, India \\ ${ }^{2}$ Assistant Professor and Head (i/c), Department of Computer Science, \\ DDE, Madurai Kamaraj University, Madurai - 625021
}

\begin{abstract}
An efficient Intrusion Detection System has to be given high priority while connecting systems with a network to prevent the system before an attack happens. It is a big challenge to the network security group to prevent the system from a variable types of new attacks as technology is growing in parallel. In this paper, an efficient model to detect Intrusion is proposed to predict attacks with high accuracy and less false-negative rate by deriving custom features UNSW-CF by using the benchmark intrusion dataset UNSW-NB15. To reduce the learning complexity, Custom Features are derived and then Significant Features are constructed by applying meta-heuristic FPA (Flower Pollination algorithm) and MRMR (Minimal Redundancy and Maximum Redundancy) which reduces learning time and also increases prediction accuracy. ENC (ElasicNet Classifier), KRRC (Kernel Ridge Regression Classifier), IGBC (Improved Gradient Boosting Classifier) is employed to classify the attacks in the datasets UNSW-CF, UNSW and recorded that UNSW-CF with derived custom features using IGBC integrated with FPA provided high accuracy of $97.38 \%$ and a low error rate of $2.16 \%$. Also, the sensitivity and specificity rate for IGB attains a high rate of $97.32 \%$ and $97.50 \%$ respectively.
\end{abstract}

\section{KEYWORDS}

Intrusion Detection, IDS, UNSW-B15, Custom Features, Feature Selection, FPA, Gradient Boost Classifier.

\section{INTRODUCTION}

The attackers are continuously developing new attack techniques to breach the user's defense security system. Most attacks use malware or social engineering to obtain user credentials and hackers are also using machine learning techniques to learn new ways to exploit networks. Earlier identification of attack vectors helps in preventing and limiting the damage caused by intrusions. Security risks can be reduced by using an effective intrusion detection system. An increase in the changing patterns of hackers shows the sign of a need for an effective Intrusion Detection System with high learning rate and reduced learning time with a high accuracy.

The feature selection (FS) aspect of detection models [1] has profound effects on their performance as some features of a large dataset of network traffic might be useless (noise), which will adversely affect the performance when they are used for training the IDS detection model. A learning algorithm is used to learn the features from the training samples by utilizing a learning algorithm in the training process. Machine learning models are trained and tested simultaneously. 
Research gaps are identified in existing works $[10,11]$ that achieve application and transport layer features among the network while failing to detect intrusions in the entire system, as well as the lack of pre-processing to identify null data, missing data, and redundant data among the datasets. With such a noisy dataset, an IDS's effectiveness can be severely limited. These missing and duplicate data that have escaped cause a high false alarm rate. A framework is proposed to overcome these limitations by introducing pre-processing to avoid noisy data, a prediction model with feature selection, and custom features as the main objectives.

In this paper, a model is proposed to detect wireless networking environment attacks using the UNSW-NB15 network intrusion detection dataset since this dataset has footprints of attack types with normal and contemporary attack activities of network traffic when compared with KDD98, KDDCup99, and NSLKDD[2]. In order to avoid noisy data in the UNSW-NB15 dataset, missing value computation, duplicate data removal, as well as unique format conversion are performed during preprocessing. The cleaned dataset was used to construct a custom feature set to eliminate the misperception during learning. The framework used metaheuristic FPA and mRMR feature selection strategy to obtain the significant features for the prediction. The significant features are obtained by evaluating the feature importance score. Novel unique custom features UNSW-CF is derived to increase the learning accuracy with reduced time. The employed supervised machine learning techniques are applied with both Custom Feature and Significant Feature to illustrate better performance while predicting. For the prediction of accurate attacks among the large dataset, the custom features set and significant features set are split into training and testing sets. This work enhances the detection rate of the IDS by employing various classifiers for the prediction of attacks like Fuzzers, Analysis, BackDoors, Dos, Exploit, Generic, Reconnainance, Shell Code, and Worms. The IGB classifier gives the best result for customized features and provides the best prediction accuracy based on the feature set.

\section{REVIEW OF LITERATURE}

The multi-stage deep learning (TSDL) model from Khan et al. [3] uses stacked auto-encoders and a soft-max classifier to detect network intrusion. To evaluate the proposed model, a comprehensive set of experiments is conducted using the KDD99 and UNSW-NB15 datasets and does not include deep learning algorithms. By integrating wrapper and filter features, Anwer et al. [4] implemented a model using UNSW-NB15 to select the feature and used Naive Bayes and J48 classifiers to detect anomalous network traffic. The limitation of this work is it identifies only a limited number of attacks. Maajid and Nalina implemented a Feature Importance (FI) score for each attribute in the UNSW-NB15 dataset using a feature reduction method based on the RF algorithm [5]. The limitation is that the computation time is high while prediction.

Zhang et al. [6] proposed IDS based on a two-stage (TS) classifier. The attributes needed for binary classification were selected using the Information Gain (IG) method. The work considers limited attacks. Using the Extreme Learning Machine (IELM) and Advanced Principal Component Algorithm (APCA), Gao et al. [7] proposed IDS based on an incremental approach. To perform optimal attack prediction, the APCA is responsible for selecting adaptively the most relevant features required by the IELM. The researchers evaluated IDS performance using UNSW-NB15. An analysis of the UNSW-NB15 intrusion detection dataset is presented in [8], which will be used for training and testing our models. Moreover, it applies a filter-based feature reduction technique using the XGBoost algorithm. Mousa Al-Akhras [13] group proposed a classification model using machine learning which automatically identifies anomaly and takes actions before an attack takes place.

Toldinascrew [9] proposed a novel methodology for detecting the intrusion using multistage deep learning recognition of the image. The feature of the network is changed into four different color 
International Journal of Computer Networks \& Communications (IJCNC) Vol.14, No.1, January 2022

channel images like red, green, blue, and alpha. Then these images are involved in the deep learning recognition using the benchmark dataset of UNSW-NB15 and BOUN Ddos. In paper [10] implemented three machine learning algorithms NB, SVM, and KNN to identify the bestsuited algorithm to detect the suspicious activities among the network by reducing the learning time and increasing accuracy. The paper [11] used the UNSW-NB15 dataset to obtain the application and transport layer features for intrusion detection to eliminate the issues like overfitting, imbalance in datasets, and curse of dimensionality. The detection of distributed collaboration scheme is proposed in the work [13] for the anomaly detection system.

IDS models need to be fast and efficient because of the changing intellectuals of intruder behavior. Researchers $[4,10,11]$ focused on analyzing a single dataset and providing research ideas based on that environment. It will take some additional time and effort to adapt their idea to other dataset. The learning problem would occur when adapting to a different environment and dataset, since training for attack pattern prediction takes more time with intrusion datasets [14]. These challenges motivated me to design a generic model for anomaly detection that reduce the learning time and adapts to different intrusion datasets within an environment to ensure high accuracy.

\section{Proposed SCheme}

In this work, the machine learning algorithm is used to improve a framework for predicting intrusions. The proposed framework addresses the environment adoption issue. The environment consists of different operating systems and hardware environments such as Linux OS, Wired/Wireless Networking Environment, and IoT Device Based Networking Environment. These frameworks were tested under various environments containing different types of datasets. The framework was already tested with the NSL-KDD dataset [12] that fulfilled the phases and provides better results. To explore the research with more than one dataset to check the framework this work chooses the UNSW-NB15 dataset. Machine learning techniques are used to predict UNSW-NB15 intrusions based on the proposed model. In the proposed model, a novel set of Custom Features (CF) is proposed to avoid the issue of learning while predicting so that enhanced prediction can be achieved using UNSW-NB15. In this way, the novel features (UNSW_CF) derived would minimize the chance of misinterpretation of feature evaluation in attack prediction. The proposed model contains five major phases for the attack prediction as Data Collection Phase, Pre-processing Phase, Construction Phase, Selection Phase, and Prediction Phase. Figure 1 shows the proposed model workflow. 


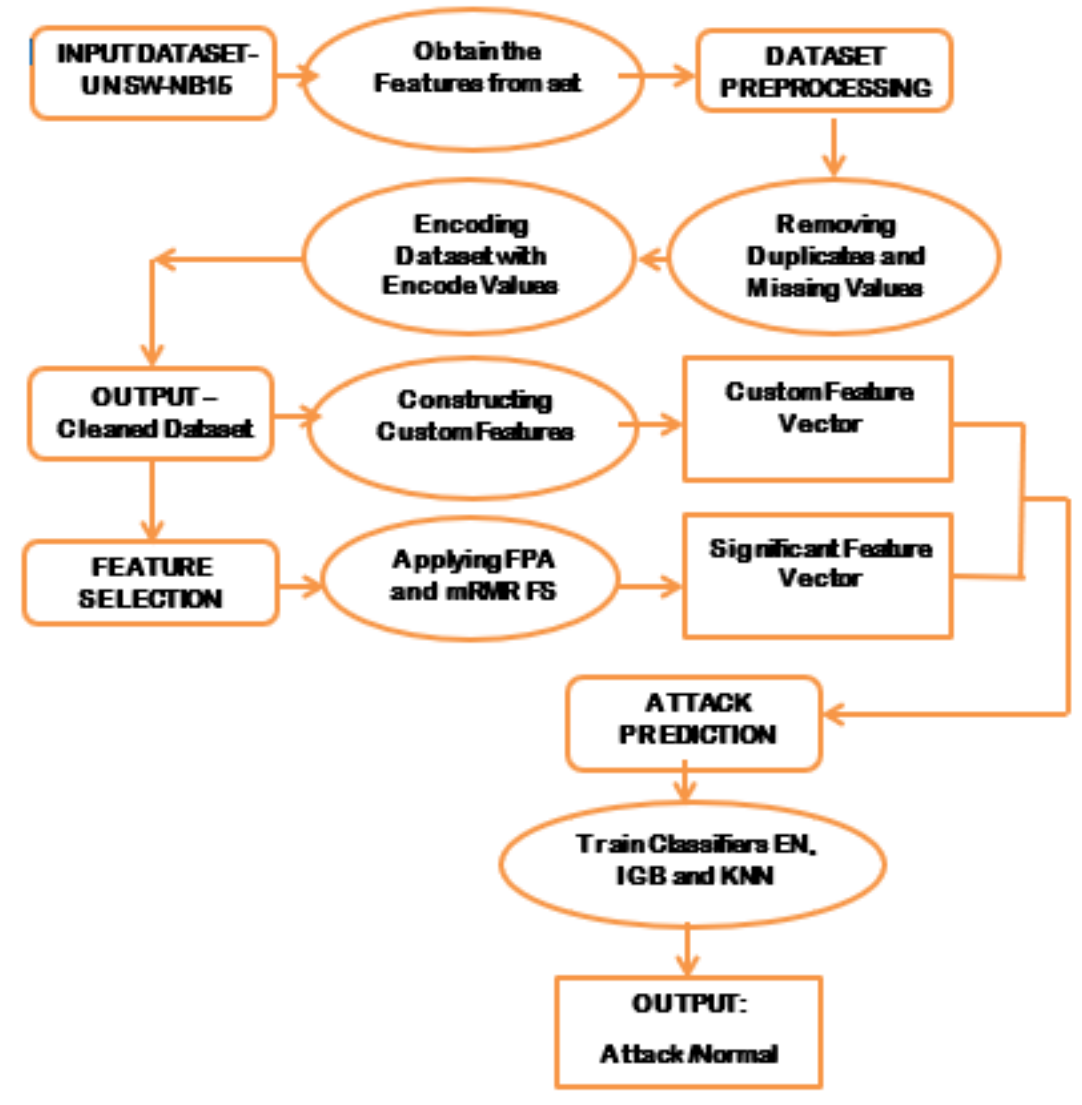

Figure 1: Proposed Workflow

\subsection{Data Collection Phase}

This section describes the collection of input datasets for the proposed framework. The section includes the UNSW-NB15 dataset description with the number of records captured with the ratio of normal and attack active records.

\subsubsection{Dataset}

The UNSW-NB15 dataset of Canberra includes nine types of modern attacks compared to the KDD dataset. UNSW-NB15 data is organized into 6 categories, namely Basic Characteristics, Flow Characteristics, Time Characteristics, Content Characteristics, Additional Generated Characteristics, and Labeled Characteristics [2]. There are 36-40 features that can be considered General Purpose Features. Connectivity features are those counting from 41 to 47 . It includes 49 features and various records of normal and attacked events with class labels of a total of 25 , 44,044 records [15].

\subsection{Pre-Processing Phase}

In the preprocessing phase, the raw data of intrusion (UNSW-NB15) is chosen for cleaning in preparation for deriving novel features. A dataset $(D)$ is pre-processed by removing duplicate columns, avoiding missing values and redundant columns, thus reducing the size of the dataset for further processing. The pre-processed dataset $\mathrm{D}_{\mathrm{C}}$ is encoded in a unique format to avoid the 
International Journal of Computer Networks \& Communications (IJCNC) Vol.14, No.1, January 2022

complexity of the evaluation of the features with various formats. Thus the dataset $\mathrm{D}_{\mathrm{C}}$ has encoded the fields with encoding values.

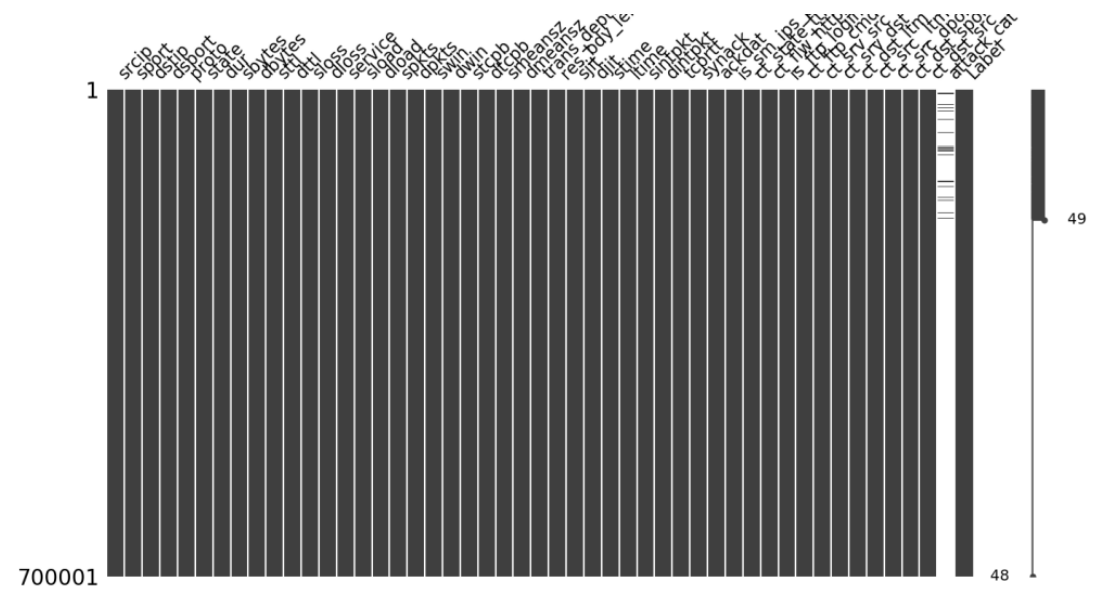

Figure 2. Missing values in UNSW Dataset

Figure 2 shows the missing values in the Dataset (D) with the size of $700001 \mathrm{X} 49$. CT_FLW_HTTP_MTHD and IS_FTP_LOGIN fields have more missing values in the Dataset.

Table 1. Encoding Value of the Dataset Fields

\begin{tabular}{lll}
\hline Features & Values & Encoded Value \\
\hline Source / Destination IP & 192.X.X.X & 0 to N \\
State & ACC,CLO & 0 to 16 \\
Protocol & HTTP, FTP & $0-134$ \\
Class & Normal / Attack & $0 / 1$ \\
\hline
\end{tabular}

The encoding value for the features of the cleaned dataset is represented in Table 1. The table depicts some of the features in the dataset is denoted with the encoded value for the unique format to avoid the complexity of deriving the novel custom features.

\subsection{Construction Phase}

This section describes novel custom features derived from the dataset $D_{C}$. From the existing basic and other datasets, fields are acquired and integrated with essential fields for the evaluation to provide Custom Features. The novel custom features set $D_{N}$ which was constructed to increase the prediction rate and plays a major part in training the classifiers with these custom features would reduce the training misinterpretation of the feature evaluation. Accordingly, the following custom features are derived:

\section{a) Unique ID (UPID)}

The UP ${ }_{I D}$ feature is derived from the given dataset features that combine to form a unique ID. The unique format for the UP $\mathrm{ID}_{\mathrm{D}}$ is:

$$
U P_{I D}=[\text { srcip, sport, dstip, dsport, proto }]
$$


International Journal of Computer Networks \& Communications (IJCNC) Vol.14, No.1, January 2022

\section{b) Proto_State}

To identify the protocol and its state, this feature uses the following format.

c) Avg_Src_Dur

$$
\text { Proto_State }=\{\text { Proto }, \text { State }\}
$$

This feature is used to calculate the average duration of bytes in a source packet. Based on the duration, Sbytes evaluates the feature.

\section{d) Avg_Dst_Dur}

$$
\text { Avg_Src } c_{\text {Dur }}=\text { Sbytes } / \text { Dur }
$$

The feature is used to determine a destination packet's average duration. Bytes and duration are utilized to compute the feature.

$$
\text { Avg_Dst } t_{\text {Dur }}=\text { Dbytes } / \text { Dur }
$$

\section{e) Avg_Dur}

This feature is derived to find the average duration of the transaction concerning the Avg_Src DurandAvg_Dst $_{\text {Dur. }}$.

$$
A v g_{\text {Dur }}=\text { Avg_SrC } C_{\text {Dur }}+\text { Avg_Dst } t_{\text {Dur }} / 2
$$

\section{f) TTL_Mean}

TTL features from the given dataset are employed to derive the mean TTL value. It is necessary to integrate the destination and source TTL's to determine the mean of TTL's.

$$
\mathrm{TTL}_{\text {Mean }}=(\mathrm{sttl}+\mathrm{dttl}) / 2
$$

\section{g) Mean of Packet Loss}

An evaluation of packet loss is based on how many packets are lost at source and destination.

$$
\text { PLoss }_{\text {Mean }}=(\text { sloss }+ \text { dloss }) / 2
$$

\section{h) Service_State}

With this feature, a service can be identified and its state can be determined.

$$
\text { Service State }=\{\text { Service, State }\}
$$

\section{i) ProtoServiceState}

In order to specify the same protocol and service, the feature is defined and its state is as follows.

$$
P S S=\{\text { Proto }, \text { Service }, \text { State }\}
$$


International Journal of Computer Networks \& Communications (IJCNC) Vol.14, No.1, January 2022

\section{j) Total Load}

Using source packet load and destination packet load, the total number of loads is calculated.

$$
\text { Total Load }=(\text { Sload }+ \text { Dload }) / 2
$$

\section{k) Total Packets}

Total source packets and destination packets are used to determine the total number of packets.

$$
\text { Total Packets }=(\text { Spkts }+ \text { Dpkts }) / 2
$$

\section{1) Bits per Load}

This feature is evaluated to determine the number of bits required for a load. The number of bits in the transaction is calculated based on how many bits there are in the total load.

$$
B P L=\text { Total Bits / Total Load }
$$

\section{m) Trans_Interval}

A feature is derived to calculate the transaction interval among the network is described. An average is calculated by averaging the start time and the last time of a transaction.

$$
\text { Stime + Ltime /2 }
$$

\subsection{Selection Phase}

This section explains the methods for selecting significant features from the dataset with and without custom features that are used to reduce dimensionality. The cleaned dataset $\mathbf{D}_{\mathbf{C}}$ is involved in the process of selection phase to obtain the significant features $\mathbf{S F}_{\mathbf{S}}$ among the set to train the classifiers for the prediction. These feature selection strategies are applied only to the cleaned dataset (not for Custom Features Set) for the analysis and obtaining of the significant features. The feature importance score is evaluated to organize the features from zero to non-zero score for the selection and elimination of the features. From the evaluation of feature selection strategies, the features from the set would obtain $1 / 3^{\text {rd }}$ of the features from the dataset for prediction.

\subsubsection{Flower Pollination Algorithm}

FPA is an algorithm inspired by nature proposed by Yang [11]. Standard FPA updates the solutions based on continuous value position updates in the search space. The initial population of Flowers " $F$ " in Flower pollination is generated through random sampling in S-dimensional space. A Flower $i$ is represented by $S$ variables, such as $F i=\left(f_{i 1}, f_{i 2} \ldots, f_{i n}\right)$. It is a problem of selecting a specific feature or not, so the solution is represented as a binary vector, where 1 indicates a selected feature and 0 if none is selected. A flower pollination algorithm, on the other hand, models the search space as a d-dimensional Boolean lattice, so the solution is updated across hypercube corners. In addition, the solution binary vectors are employed since it is the problem of whether the given feature should be selected or not, and 0 otherwise. To build this binary vector: 
International Journal of Computer Networks \& Communications (IJCNC) Vol.14, No.1, January 2022

$$
\begin{gathered}
S\left(x_{i}^{j}(t)\right)=\frac{1}{1+e^{-x_{i}^{j}(t)}} \\
x_{i}^{j}(t)=\left\{\begin{array}{c}
1, \text { if } S\left(x_{i}^{j}(t)\right)>\sigma \\
0, \text { otherwise }
\end{array}\right.
\end{gathered}
$$

In which $x_{i}^{j}(t)$ denotes the new pollen solution, $I$ with the $j$ th feature $\mathrm{r}$, where $i=1,2, \ldots m$ and $j=1,2, \ldots d$, at the iteration $t$ and $\sigma \mathrm{U}(0,1)$. In both classification and regression problems, FPA is used for feature selection. In the case of a vector with $\mathrm{N}$ features, the number of features to select would be $2 \mathrm{~N}$, which is the range of features to be searched exhaustively. An adaptive search area is mapped to find the best feature subset using intelligent optimization. A common objective in literature is to select a feature subset that has the smallest prediction error. When dealing with classification problems, the general fitness function is to maximize accuracy and select the minimum number of features while still selecting the maximum amount of features.

\subsubsection{MRMR}

The mutual information-based mRMR method is a method for selecting features. A high correlation between the features and the class is achieved when mRMR is used and a low correlation between the features themselves. This method has the advantage of being computationally efficient and generalizable to different machine learning models. The mRMR method differs from other filter methods in that it can effectively reduce redundant features while preserving the relevant features. As many important features are correlated and redundant, the $m$ best features are not the best " $\mathrm{m}$ " features [5].

When selecting features for mRMR, both relevance and redundancy are taken into the interpretation. MI mutual information between features is used as a redundancy measure. When MI is high, it means that both features are duplicating a lot of information that is, there are redundancies between them. A redundancy value lower than zero indicates a better criterion for feature selection. Utilizing redundancy means selecting the feature that has the lowest MI among all other features. MI between the feature and the target activity is used to determine the relevance measure. A small MI value indicates that the correlation between the feature and the target activity is weak. In contrast, a larger MI value indicates that the feature contains more information to classify activity. In the work, given that the input data $D S$ with ' $N$ ' number of inputs that have ' $f$ ' number of features in the set $F_{S}$, with the subset $\mathrm{F}_{\mathrm{S} i}=\{\mathrm{f} 1, \mathrm{f} 2, . ., \mathrm{fi}\}$. MI is evaluated to find out the dependencies among the feature set FS is computed using the following Equation:

$$
\operatorname{MI}\left(f_{i}, f_{j}\right)=\sum_{i, j} \frac{\operatorname{prob}\left(f_{i}, f_{j}\right)}{\operatorname{prob}\left(f_{i}\right) \operatorname{prob}\left(f_{i}\right)}
$$

Assume $\mathrm{F}_{\mathrm{S} i}$ is a given set of features and $\mathrm{C}$ is a target class. The redundancy of $\mathrm{F}_{\mathrm{S} i}$ is measured by:

$$
F_{R e d}\left(F_{S i}, C\right)=\frac{1}{\left|F_{S i}\right|} \sum_{i, j \in S_{i}} M I\left(f_{i}, f_{j}\right)
$$

Redundancy can result from the selection of features that are consistent with maximizing relevance, meaning they are interdependent. Whenever two features are extremely interdependent, their class-discriminatory effects don't change much if one is removed. As a result, the highly relevant features are classified based on the highest relevancy rating of the 
evaluated feature $f_{i}$ to create a feature subset $S_{i}$. To compute the Significant Feature Selection Set $\mathbf{S F}_{\mathbf{S}}$, the weighting process is integrated to prevent redundant values from the evaluated feature subset $S_{\mathrm{i}}$.

The process of weighting is included utilizing MI for every pair of features from every feature subset $\mathrm{F}_{\mathrm{Si}}$. Then the assessed $\mathrm{MI}$ is used in evaluating up the features to eliminate the redundant features from $\mathrm{F}_{\mathrm{Si}}$ to make minimum redundant feature set is given by using,

$$
F_{R e l}\left(F_{S i}, C\right)=\frac{1}{\left|S_{i}^{2}\right|} \sum_{i, j \in S_{i}} M I\left(f_{i}, f_{j}\right)
$$

The above minimal redundancy (Minimum Redundancy) condition can be added to select mutually exclusive features. The mRMR aims to minimize redundancy while maximizing relevance when ranking features. This operation is implemented by an operator $\Phi$

$$
\max \Phi\left(F_{\text {Rel }}, F_{\text {Red }}\right)=F_{\text {Rel }}-F_{\text {Red }}
$$

\subsection{Prediction Phase}

The attack is predicted by employing machine learning after a feature selection process to obtain significant features $\mathrm{SF}_{\mathrm{S}}$ that will increase prediction accuracy. This topic describes how machine learning is used to identify attacks from network packets based on the predicted attack. The prediction model should be evaluated with suitable methods. The main aim is to train a model for prediction by validating with the testing set. The work splits the dataset into Training and Testing sets to avoid independent sets and obtain more dependent ones. The set of $80 \%$ is split for training the model and the set of $20 \%$ of data is assigned for testing the prediction model. During training the classifiers, the work employs K-Fold Validation to perform the process of fitting the data to the model. The following classifiers are used for the attack prediction.

\subsection{1. $\quad$ ENC (Elastic Net Classifier)}

Combined ridge and lasso regression models form the basis of Elastic Net (EN). A lasso or ridge regression is also typically used when predictors exceed observations, but EN overcomes some of the limitations of both. Additionally, EN often encourages "grouping," in which strongly correlated predictors are included or excluded together.

In this technique, the intermediate layer of the network graph is added as an auxiliary output, and the network is trained against the joint loss over all layers. With this simple concept of adding intermediate outputs, the Elastic Net could seamlessly switch between different levels of computational complexity while simultaneously achieving improved accuracy when the computational budget was high. Once the algorithm is built to outperform execution speed, this methodology can be useful for big datasets. There are three types of regression modeling: linear, logistic, and multinomial. A prediction is an objective, as well as the minimization of the prediction error, both in terms of model choice as well as estimation.

For a given $\lambda, \mathrm{Y}$ is the response variable, and $\mathrm{X}$ measures the predictors. Taking a dataset $\left(\mathrm{x}_{\mathrm{i}}, \mathrm{y}_{\mathrm{i}}\right)$, $\mathrm{i}=1, \ldots, \mathrm{N}$, the elastic net approach provides the following solution:

$$
\min _{\beta_{0}, \beta}\left[\frac{1}{2 N} \sum_{i=1}^{N}\left(y_{i}-\beta_{0}-x_{i}^{T} \beta\right)^{2}+\lambda \mathrm{P}_{\alpha}(\beta)\right]
$$


Based on the value of $\alpha$, the elastic net penalty is calculated as follows:

$$
\begin{aligned}
& P_{\alpha}(\beta)=(1-\alpha) \frac{1}{2}\|\beta\|_{l_{2}}^{2}+\alpha\|\beta\|_{l_{1}} \\
& P_{\alpha}(\beta)=\sum_{j=1}^{p}\left[\frac{1}{2}(1-\alpha) \beta_{j}^{2}+\alpha\left|\beta_{j}\right|\right]
\end{aligned}
$$

Where $\mathrm{P}_{\alpha}(\beta)$ is the elastic-net consequence to find the negotiation among the ridge regression $\alpha=$ 0 and Lasso penalty $\alpha=1$, that acquires the minimum limit that lies between ' $\mathrm{t}$ ', $\mathrm{P}_{\alpha}(\beta)<\mathrm{t}$. The parameter $\mathrm{P}$ is compared to $\mathrm{N}$ would determine the correlation of the predictors that chooses whether the ridge or lasso that shrink the coefficients of correlated predictors and indifferent to correlated predictors respectively. If the value of $\alpha$ is close to 1 then the determination of correlation between $\lambda$ and $\alpha$. An $1_{\mathrm{q}}(1<\mathrm{q}<2)$ penalty term could also be considered for prediction would produce the prediction result.

Table 2. Tuning Parameters for EN

\begin{tabular}{lll}
\hline Parameters & Default Value $(\boldsymbol{d} f)$ & Improved Value $(\boldsymbol{I})$ \\
\hline Alpha $(\boldsymbol{\alpha})$ & 1.0 & 6.0 \\
L1_Ratio (li) & 0.5 & 0.7 \\
Maximum Iteration (MI) & 1000 & 5000 \\
Selection (SL) & Cyclic & Random \\
N-Splits (K-Fold) (N-S) & 10 & 20 \\
Random State (RS) & 1 & 7 \\
\hline
\end{tabular}

The Elastic Net Classifiers value has been updated to "6" as shown in Table 2. The penalty is calculated by adding L1 and L2 together as a method for calculating the L1_Ratio (0.7). The maximum number of iterations is set at 5000. In the default setting, the selection is set to random, but instead of looping over features Cyclic by default, a random coefficient is updated every time. There are twenty K-folds, and each K-fold has seven different random states for validation.

\subsection{2. $\quad$ KRRC (Kernel Ridge Regression Classifier)}

Kernel Ridge Regression (KRR), one of the most popular types, uses kernel methods. A kernelbased approach is particularly useful when there is a nonlinear structure to the data [6]. Compared to other sophisticated methods such as SVM, KRR is simpler and faster to train with its closed-form solution. As a classifier, KRR is considered to be strong. The problem here is that this yields an unstable KRR classifier, suitable for ensemble methods. By training it with only a subset of the whole training set, the method achieves this. Kernel Ridge Classification (KRRC) is based primarily on transforming the original samples into higher-dimensional Hilbert spaces and then applying regression techniques to them. By using a kernel trick, you can represent a nonlinear curve as lying on a plane. It can be denoted as follows $\phi: \mathrm{X} \rightarrow \mathrm{F}$. In kernel ridge regression, the aim is to find $\widehat{\alpha}_{l}$ the minimum residual error is as follows:

$$
\widehat{\alpha}_{\imath}=\underset{\alpha_{i}}{\arg \min }\left\|\varnothing(x)-\emptyset\left(X_{i}\right) \alpha_{i}\right\|_{2}^{2}+\lambda\left\|\alpha_{i}\right\|_{2}^{2}
$$

It is possible to compute the regression parameter vectors by 
International Journal of Computer Networks \& Communications (IJCNC) Vol.14, No.1, January 2022

$$
\widehat{\alpha}_{\iota}=\left(\emptyset^{T} \emptyset\left(X_{i}\right)+\lambda \mathrm{I}\right)^{-1} \emptyset^{T}\left(X_{i}\right) \emptyset(x)
$$

Kernel ridge regression extends linear regression into non-linear and high-dimensional space using the "kernel trick". The data $x_{i}$ in $X$ is replaced with the feature vectors: $x_{i} \rightarrow \Phi=\Phi\left(x_{i}\right)$ induced by the kernel where $\mathrm{K}_{\mathrm{ij}}=\mathrm{k}\left(\mathrm{x}_{\mathrm{i}}, \mathrm{x}_{\mathrm{j}}\right)=\Phi\left(\mathrm{x}_{\mathrm{i}}\right) \Phi\left(\mathrm{x}_{\mathrm{j}}\right)$. As a result, the predicted class label for an example $\mathrm{x}$ is:

$$
Y^{T}(k+\lambda I)^{-1} k
$$

Where $\mathrm{k}=\left(\mathrm{k}_{1}, \ldots, \mathrm{k}_{\mathrm{N}}\right)^{\mathrm{T}}, \mathrm{k}_{\mathrm{n}}=\mathrm{x}_{\mathrm{n}} \cdot \mathrm{x}$ and $\mathrm{n}=1, \ldots, \mathrm{N}$. Kernel ridge regression uses Kernel functions such as Gaussian, linear, or polynomial that do not access feature vectors $\Phi(\mathrm{x})$. It translates classification problems into regression problems with class labels since KRR is naturally suited to handling regression problems. This is achieved by coding the output target $\mathrm{Y}$ as $0-1$. As a result of having $\mathrm{N}$ samples for ' $\mathrm{m}$ ' classes, the output $\mathrm{Y}$ is a " $\mathrm{N} \mathrm{x} \mathrm{m}$ " matrix generated according to the following equation:

$$
Y_{i j}=1, \text { if } i^{\text {th }} \text { sample belongs to Class, otherwise } 0
$$

Table 3. Tuning Parameters for KRR

\begin{tabular}{lll}
\hline Parameters & Default Value $(\boldsymbol{d} f)$ & Improved Value $(\boldsymbol{I})$ \\
\hline Alpha (A) & 1.0 & 5.0 \\
Maximum Iteration (MI) & None & 100 \\
Class Weight (CW) & Dict & Balanced \\
Solver (S) & Auto & LSQR \\
N-Splits (K-Fold) (N-S) & 10 & 20 \\
Random State (RS) & 1 & 7 \\
\hline
\end{tabular}

To improve the conditioning process for the problem and reduce variance, the Alpha value of the proposed Ridge Classifier will be updated to "5" $(I(A))$ to improve efficient algorithms is described in Table 3. To ensure accuracy, the conjugate gradient solver can only execute 100 as Maximum iterations. When the weight for a class is declared "Balanced" mode, it is adjusted with the values of y inversely proportional to the class frequency. The least-squares routine is set in the computation routine solver. Each of the twenty K-folds will make a different validation by choosing seven distinct random states.

\subsubsection{IGBC (Improved Gradient Boosting Classifier)}

Gradient boosts can be used as regression and classification algorithms. The method is based on the combination of Gradient Descent and Boosting. A forward stage-by-stage approach to fitting ensemble models is involved. A generalization of an adaptive boosting scheme that can handle a variety of loss functions for gradient boosting is proposed. The gradient boosting (GB) algorithm sequentially builds new models out of weak models to minimize the loss function of each new model. Loss functions make each model fit the observations more accurately, resulting in increased accuracy. However, boosting must eventually stop to prevent the model from becoming overfit. It is possible to set stopping criteria based on the accuracy of predictions or the number of models created.

By optimizing an objective loss function, the ensemble model is built and generalized in a stagewise fashion. GB techniques build their models iteratively by analyzing the previous loss function of negative gradients. Loss function minimization is a crucial component of ML and 
must be optimized. Therefore, the loss function shows the difference between a predicted and target output. Loss function values below a certain threshold mean a high prediction or classification result. A Gradient of Loss Function is the result of the loss function decreasing sequentially and iteratively along a specific direction. In supervised classification problems, the objective is to find an approximation function $\mathrm{O}^{\prime}(\mathrm{x})$ to fit the $\mathrm{O}(\mathrm{x})$. Based on a loss function, we define the following approximation function, $\mathrm{L}(\mathrm{y}, \mathrm{O}(\mathrm{x}))$ :

$$
\hat{O}(x)=\underset{O(x)}{\operatorname{argmin} L}(y, O(x))
$$

Where $\mathrm{O}$ represents the weak learners $\left(C_{i}(x)\right)$ with weights $\left(w_{\mathrm{i}}\right)$ in a linear combination; The loss function of an input vector is minimized by $\mathrm{O}^{\prime}$. Thus, the GB sets a constant function, $\mathrm{O}_{0}(\mathrm{x})$ as:

$$
O_{0}(x)=\underset{w}{\operatorname{argmin} L} \sum_{i=1}^{n} L\left(y_{i}, w\right)
$$

If a decision tree is chosen as an estimator, gradient boosting will be chosen as a good algorithm to use; it is a classifier that can be applied to many problems in humanities. In a previous post, we discussed different boosting algorithms. Gradient boosting is regarded as the most effective algorithm among these options. While GB mainly relies on convex loss functions, it is capable of using a wide range of loss functions. Furthermore, GB is also capable of solving classification and regression problems. When dealing with classification problems, the log loss function is employed as the objective function.

Table 4. Parameter Tuning for IGB (Proposed)

\begin{tabular}{lll}
\hline Parameters & Default Value $(\boldsymbol{d} \boldsymbol{f})$ & Improved Value $(\boldsymbol{I})$ \\
\hline N-Estimators (N-E) & 100 & 200 \\
Learning Rate (LR) & 0.1 & 2.0 \\
Max Depth (MD) & 3 & 9 \\
CCP_Alpha (CCPA) & 0 & 0.5 \\
Maximum Leaf Nodes (MLN) & None & 10 \\
Verbose (V) & 0 & 1 \\
N-Splits (K-Fold) (N-S) & 10 & 20 \\
Random State (RS) & 1 & 7 \\
\hline
\end{tabular}

There are two kinds of parameters in IGB (Improve Gradient Boost). One is the Default value, and the other is the improved value. An improved classifier uses tuned classifier parameters. It improves the accuracy of the classifier. In this IGB algorithm, the N-Estimator performs boosting stages and is set to 100 by default and 200 by tuning is shown in Table 4 . It helps to avoid overfitting when the Learning Rate $\left(\mathrm{L}_{\mathrm{R}}\right)$ is low because a slower learning rate increases overfitting risk, Where, $d f\left(\mathrm{~L}_{\mathrm{R}}\right)=0.1 \& I\left(\mathrm{~L}_{\mathrm{R}}\right)=2$. With Maximum Depth, the value Nine is selected and the Best Performance is selected (Values 1-9), that identify based on the lowest error. The CCP-Alpha pruning method (0.5) used for minimizing the cost of complex pruning is used to avoid overfitting. The maximum number of leaf nodes determines how long a tree will grow; the best number (10) is defined as a relative reduction in impurities. This K-fold is split into twenty and will make a different validation using seven different random states. 


\section{EXPERIMENTS AND RESULTS}

To reduce the dimensionality of the cleaned set, UNSW is incorporated with the feature selection strategy based on the features accumulated from the given dataset. The significant feature set $\mathrm{SF}_{\mathrm{S}}$ is obtained for the prediction model learning based on the feature importance score. After applying the feature selection algorithm, the dimensionality reduction of the features set is $33 \%$ of the given dataset using the importance score of features. The proposed method FPA provides significant features with high importance score for the prediction model. In the prediction phase, the obtained significant features $\mathrm{SF}_{\mathrm{S}}$ set is employed to train the model for the prediction. In this section are illustrated the evaluation results based on the performance metrics of accuracy, error rate, sensitivity, and specificity of the classification techniques.

Accuracy: It is a classifier performance measure to evaluate the total number of predictions made proportional to the total number of predictions. Figure 3 (a) shows that the proposed Gradient Boost algorithm predicts the UNSW and UNSW_CF with accuracy is high and the amount of error is low.

$$
A c c=\frac{P_{\text {Correct }}}{P_{\text {Total }}} * 100
$$

Where $P_{\text {Correct }}$ represents the number of correct predictions of the classifier and $P_{\text {Total }}$ denotes the total number of predictions while prediction.

Error Rate: The performance rate which is evaluated to compute the misclassified instances among the total instances is shown in Figure 3 (b).

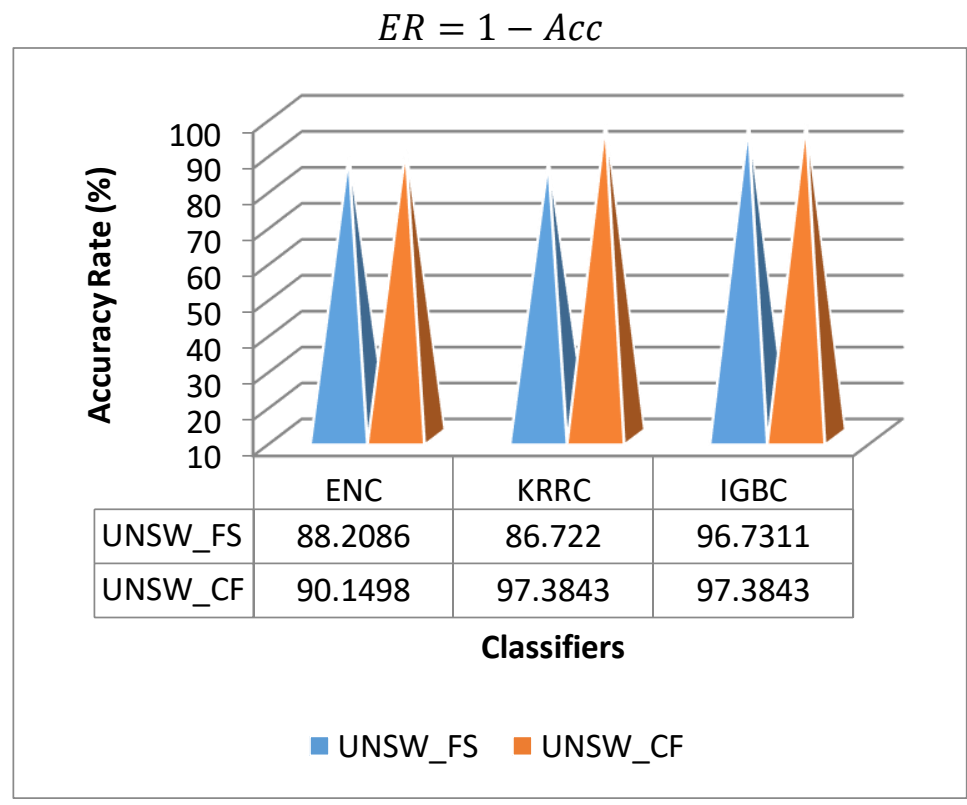


International Journal of Computer Networks \& Communications (IJCNC) Vol.14, No.1, January 2022

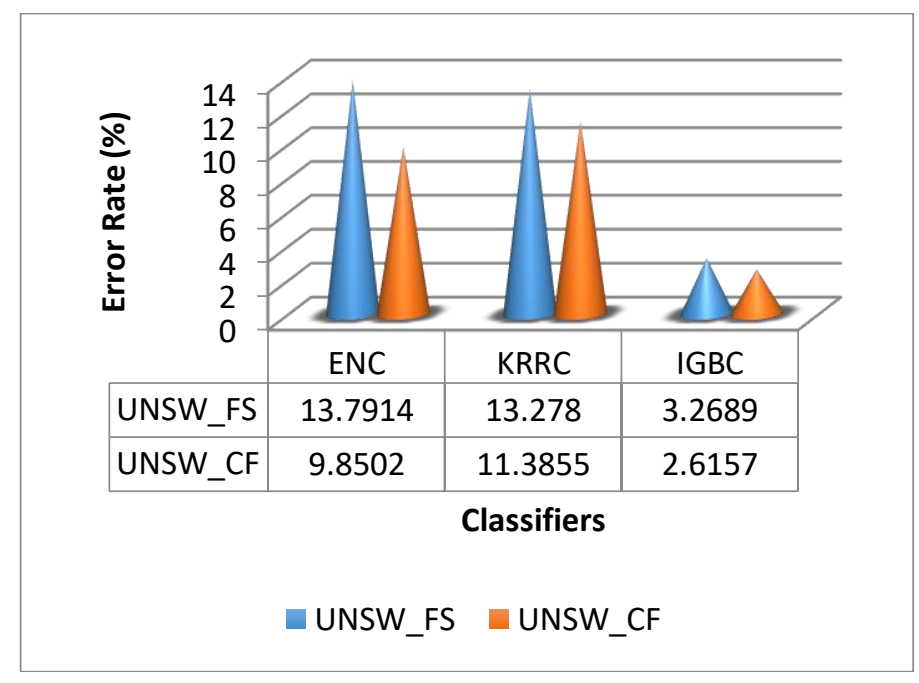

Figure 3. (a) Accuracy Rate (b) Error Rate for the Classification Techniques

Sensitivity: The evaluation metrics that used to measure the prediction of the true positive (TP) of the set by the prediction model. As shown in Figure 4, the classification techniques used for UNSW and UNSW_CF are sensitive and specific.

Specificity: The prediction of the actual number of true negative (TN) of the given set of the model.

$$
\begin{aligned}
& \text { Sensitivity }=\frac{T P}{T P+F N} \\
& \text { Specificity }=\frac{T N}{T N+F P}
\end{aligned}
$$

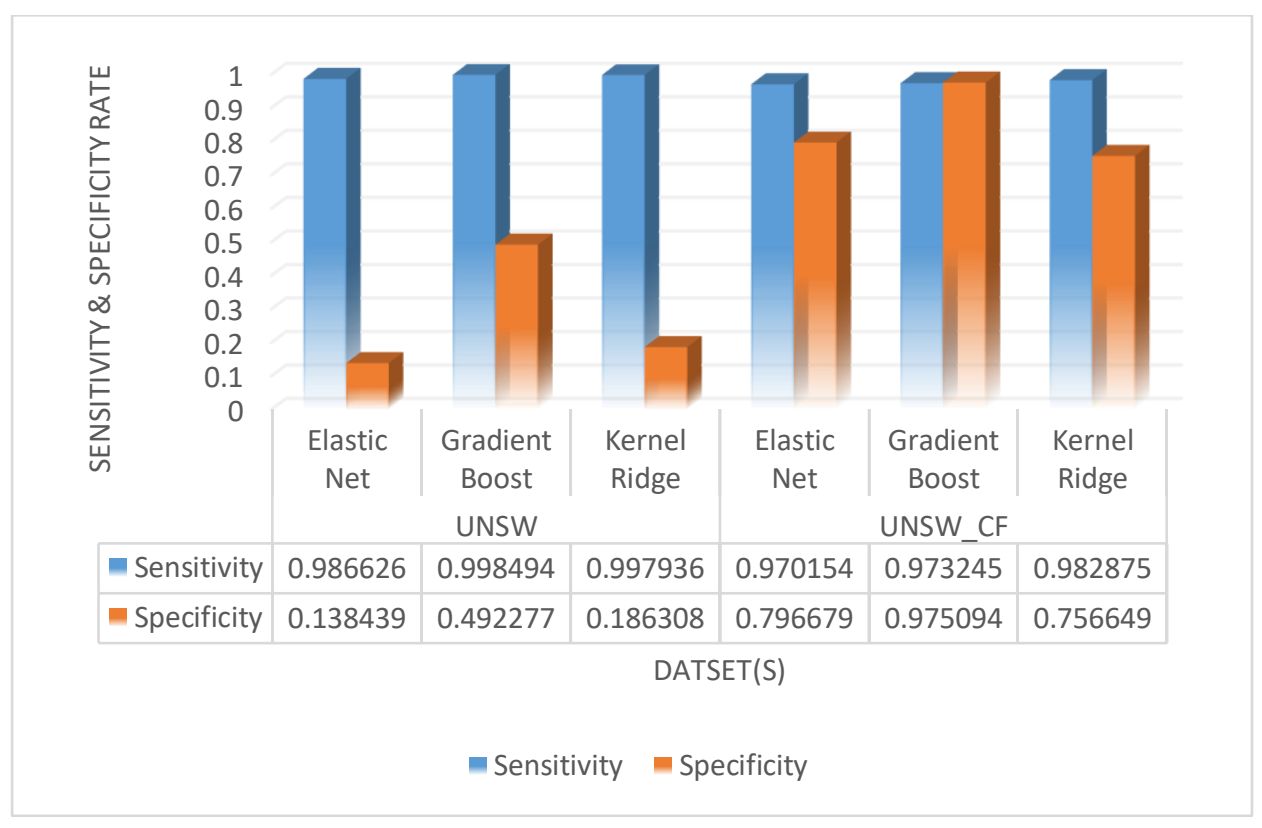

Figure 4. Sensitivity and Specificity for Prediction 
International Journal of Computer Networks \& Communications (IJCNC) Vol.14, No.1, January 2022

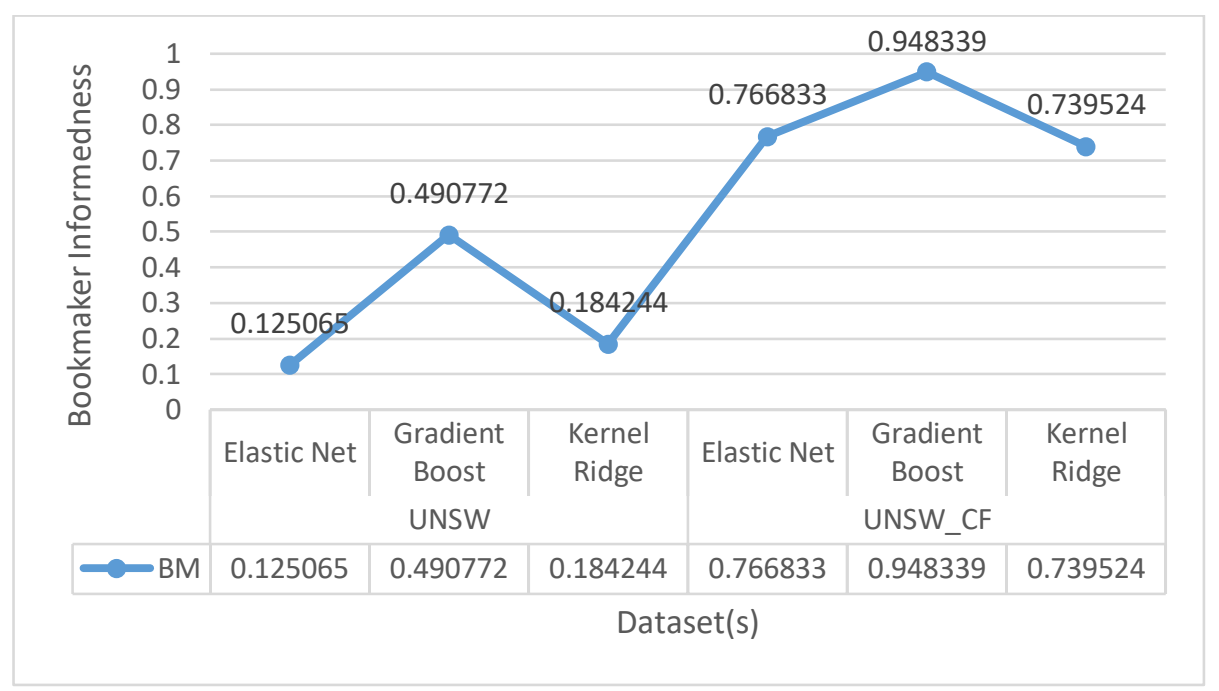

Figure 5. Bookmaker Informedness

$$
B M=T P R+T N R-1
$$

The Bookmaker Informedness (BM) is evaluated with the True Positive Rate (TPR) and True Negative Rate (TNR) that provides the worst and best values that range from -1 to +1 . The BM evaluation provides high for the proposed method Gradient Boost is shown in Figure 5.

$$
\begin{aligned}
& L R P=\text { Sensitivity } /(1-\text { Specificity }) \\
& L R N=(1-\text { Sensitivity }) / \text { Specificity }
\end{aligned}
$$

Figure 6 shows the likelihood ratio positive (LRP) and likelihood ratio negative (LRN). As the figure illustrates, the LRP provides a high rate, while the LRN provides a low rate, so the target condition has a high likelihood ratio

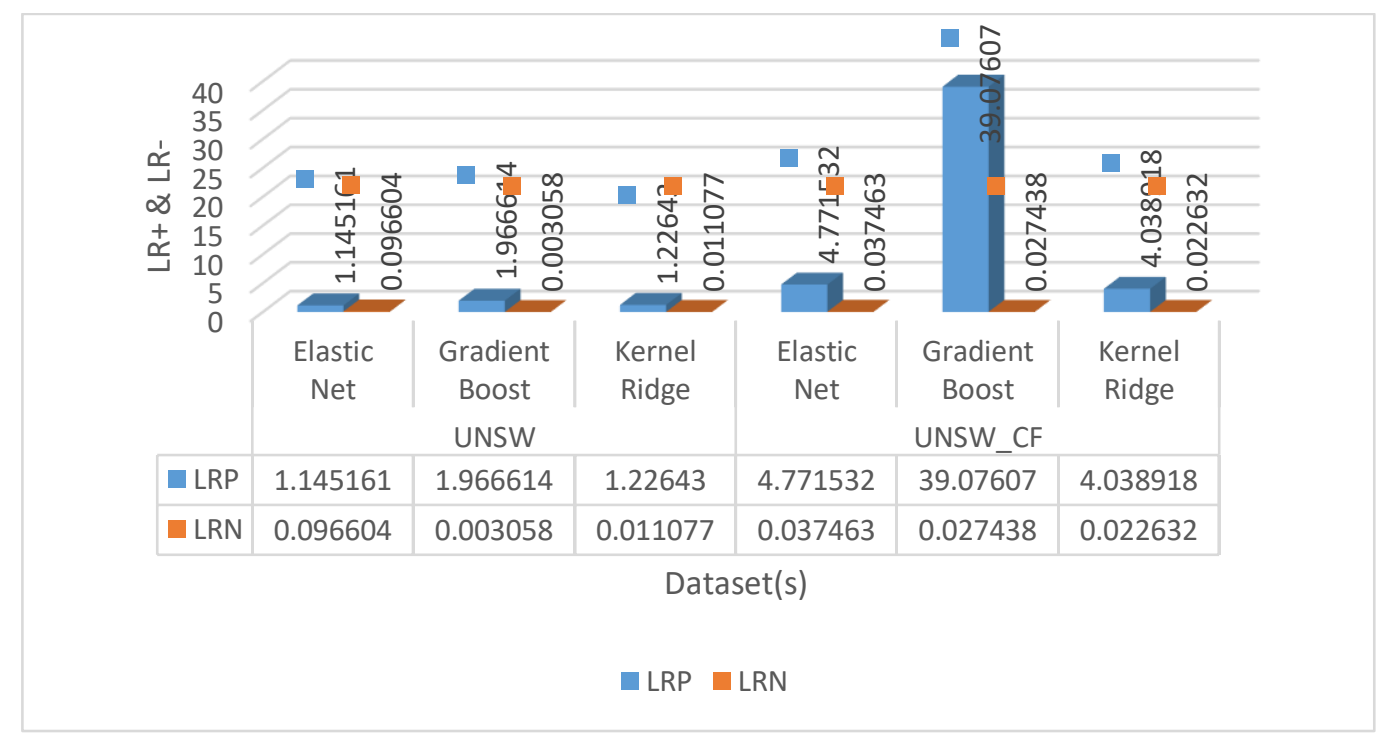

Figure 6. Likelihood Ratio Positive and Likelihood Ratio Negative 
Diagnostic odds ratios (DORs) indicate a test's effectiveness. The LLR is defined as the ratio of the probability of a positive test with an attack to the probability of a positive test without an attack, as shown in Figure 7.

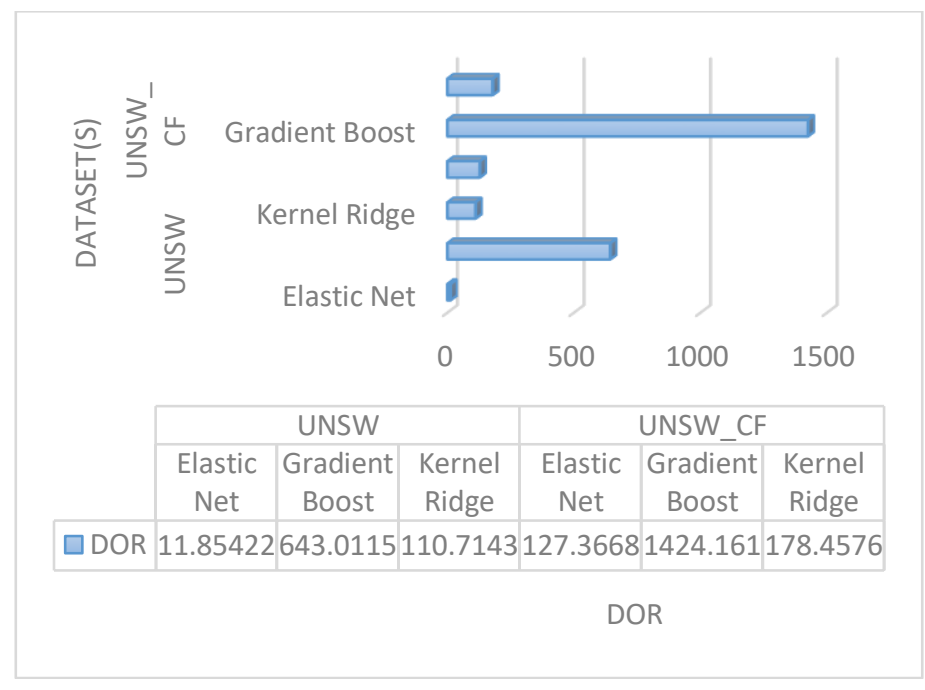

Figure 7. Diagnostic Odds Ratio (DOR)

\section{Conclusion}

This work introduces a framework for intrusion detection that deals with attack identification and prediction using machine learning strategies in the UNSW-NB15 dataset. The proposed model that employs the UNSW dataset for the attack prediction which includes the novel custom feature derivation for the effective prediction is implemented. The model deals with both UNSW and UNSW_CF features for the prediction using machine learning. The preprocessing phase is employed to clean the raw dataset to avoid duplicate records, missing columns, and null values from the dataset. The cleaned dataset is then analyzed to evaluate the custom features for the prediction. This work uses feature selection strategies such as FPA and mRMR for dimensionality reduction by selecting the most significant features which would increase the accuracy of the prediction while detection. Then the classification techniques (EN, IGB, and KRR) are applied to the significant feature subset for the attack detection. The performance results show that the proposed technique IGB gives better results for performance metrics like accuracy, error rate, sensitivity, specificity, DOR, LRP, and LRN. The framework would be included with various algorithms for the exploration. Also, the framework can be extended with the inclusion of different datasets and algorithms for enhancement.

\section{CONFLICT OF INTEREST}

The author declare no Conflicts of Interest.

\section{REFERENCES}

[1] T. A. Tchakoucht and M. Ezziyyani, (2018),"Building A Fast Intrusion Detection System For HighSpeed- Building A Fast Intrusion Detection System For High-Speed- Networks : Probe and DoS Attacks Detection", In Proc. of the First International Conference On Intelligent Computing in Data Sciences, Vol. 127, pp. 521-530. 
International Journal of Computer Networks \& Communications (IJCNC) Vol.14, No.1, January 2022

[2] Moustafa, N., Slay, J., 2015, "Unsw-nb15: a comprehensive data set for network intrusion detection systems (unsw-nb15 network data set)", Military communications and information systems conference (MilCIS), IEEE, pp. 1-6.

[3] F. A. Khan, A. Gumaei, A. Derhab, and A. Hussain, (2019), "TSDL: A two-stage deep learning model for efficient network intrusion detection”, IEEE Access, Vol. 7, pp. 30373-30385.

[4] H. M. Anwer, M. Farouk, and A. Addel-Hamid, (2018), "A Framework for Efficient Network Anomaly Intrusion Detection with Features Selection", In: Proc. of the 9th International Conference on Information and Communication Systems (ICICS), pp. 157-162.

[5] Khan NM, Negi A, Thaseen, (2018), "Analysis on improving the performance of machine learning models using feature selection technique", In: International conference on intelligent systems design and applications, Springer, pp. 69-77.

[6] Zong W, Chow Y-W, Susilo W., (2018), "A two-stage classifier approach for network intrusion detection", International conference on information security practice and experience. Springer, pp. 329-340.

[7] Gao J, Chai S, Zhang B, Xia Y., (2019), "Research on network intrusion detection based on incremental extreme learning machine and adaptive principal component analysis", Energies 2019, Vol. 12, No. 7.

[8] Sydney M. Kasongo and Yanxia Sun, (2020), "Performance Analysis of Intrusion Detection Systems Using a Feature Selection Method on the UNSW-NB15 Dataset", Journal of Big Data. Springer Open, pp. 1-20.

[9] Toldinas, J. Ven`ckauskas, A. Damaševi cius, R.; Grigaliunas, Š. Morkevǐcius, N. Baranauskas, E., (2021), "A Novel Approach for Network Intrusion Detection Using Multistage Deep Learning Image Recognition”, Electronics 2021, Vol. 10, No. 1854, https://doi.org/10.3390/ electronics10151854.

[10] Agarwal A, Sharma P, Alshehri M, Mohamed AA, Alfarraj O., (2021), "Classification model for accuracy and intrusion detection using machine learning approach”, PeerJ Computer Science, DOI 10.7717/peerj-cs.437.

[11] Ahmad, M., Riaz, Q., Zeeshan, M., (2021), "Intrusion detection in the internet of things using supervised machine learning based on application and transport layer features using UNSW-NB15 data-set", Journal of Wireless Communication Network 2021, Vol. 10 , https://doi.org/10.1186/s13638-021-01893-8.

[12] D.V. Jeyanthi, Dr. B. Indrani, (2021), "Intrusion Detection System intensive on Securing IoT Networking Environment based on Machine Learning Strategy", Springer, Proceedings of the $5^{\text {th }}$ International Conference on Intelligent Data Communication Technologies and Internet of Things (ICICI-2021).Lecture Notes on Data Engineering and Communications Technologies, DOI : 10.1007/978-981-16-7610-9

[13] Mousa Al-Akhras, Mohammed Alawairdhi1 Ali Alkoudari and Samer Atawneh, "using machine learning to build a classification model for iot networks to detect attack signatures", International Journal of Computer Networks \& Communications (IJCNC), https://ijcnc.com/2020/12/12/ijcnc-07-15/

[14] Tran Hoang Hai, Le Huy Hoang, and Eui-nam Huh, (2020), "Network Anomaly Detection Based On Late Fusion Of Several Machine Learning Algorithms", International Journal of Computer Networks \& Communications (IJCNC), Vol.12, No.6, pp. 117-131, DOI: 10.5121/ijcnc.2020.12608

[15] Nour Moustafa and Jill Slay, "The evaluation of Network Anomaly Detection Systems: Statistical analysis of the UNSW-NB15 data set and the comparison with the KDD99 data set" ,Information Security Journal: A Global Perspective, Taylor \& Francisdoi:10.1080/19393555.2015.1125974

\section{AUTHORS}

1. D.V. Jeyanthi, working as Assistant Professor in Department of Computer Science, Sourashtra College, Madurai. Doing research work in Network Security and Intrusion Detection System.

2. Dr. B. Indrani, working as Assistant Professor and Head(i/c) in Department of Computer Science in DDE Section, Madurai Kamaraj Univerisity. Madurai. Highly interested in doing research in network security, cryptography and big data. Has published various research papers in international scopus, web of science, springer, referred journals. 